\title{
COVID-19 Severity among Cancer-COVID Patients with Different Types of Cancer: A Case Series of Five Patients
}

\author{
Budhadev Baral ${ }^{1 *}$, Kartik Muduli, Shweta Jakhmola ${ }^{1}$, Sabyasachi Parida ${ }^{3}$, \\ Selvakumar Elangovan², Nirmal Kumar Mohakud ${ }^{4}$, Hem Chandra Jha ${ }^{1}$
}

${ }^{1}$ Department of Biosciences and Biomedical Engineering, Indian Institute of Technology Indore, India. ${ }^{2}$ School of Biotechnology, KIIT deemed University, Odisha, India. ${ }^{3}$ Department of Oncology \& Onco-Surgery, Kalinga Institute of Medical sciences, KIIT deemed University, Odisha, India. ${ }^{4}$ Department of Paediatrics, Kalinga Institute of Medical sciences, KIIT deemed University, Odisha, India.

\begin{abstract}
Background: People with malignancy are under increased threat in the current COVID-19 pandemic. It is crucial to study the severity of COVID-19 in different cancer type as the microenvironments are different. Besides, the effect on specific organs should be studied to understand the long-term consequences of both the diseases and its treatment. Methods: In our retrospective case series, we are presenting degree of severity in 5 cancer-COVID patients based on their clinical characteristics. All the patients were RT-PCR confirmed COVID-19 patients with malignancy. The demographic and clinical data of the patients were retrieved from the hospital database. Results: The cancers include; stomach (1), bladder (2), glioma (3), lungs (4) and hard palate cancer (5). Patient-4 and 5 were receiving chemotherapy when they got infected and had highly dysregulated liver function and higher CT severity score (19/25) compared to the nonrecipients. In patient-2 inflammatory and other haematological parameters we highly dysregulated suggesting a possible bottle neck of older age. Interestingly in the patient-3 the COVID-19 markers like lymphocyte, neutrophil and platelet levels were close to or within the normal limits unlike other patients. Conclusions: All the patents included in our report died during hospitalization. The patients receiving chemotherapy had possible impaired liver function and died early compared to the nonrecipients. The characteristic COVID-19 markers like lymphopenia, neutrophilia and thrombocytopenia did not occur in the glioma patient. The effect of cancer-COVID and its treatment on specific organs like the liver and kidney need to be studied during treatment and follow-up.
\end{abstract}

Keywords: Cancer-COVID, SARS-CoV-2, Oncotherapy, Inflammation, Severity

\section{Introduction}

The COVID-19 pandemic continues to be a threat to the immunocompromised population worldwide. Cancer is a deadly disease and the affected persons are often immunocompromised. The co-occurrence of SARS-CoV-2 infection with cancer increases the severity and often results in a higher death rate [1]. Multiple organ dysfunction is more common in patients with cancer than without it, in the case of COVID-19 [1]. However, the severity of COVID-19 in the chemotherapy receiving population is still debatable [2]. Coexisting morbidities are also important contributing factors in determining disease severity and should be considered during the treatment of COVID-19 [3]. These observations have changed the way of cancer treatment and care in the COVID-19 era. Studies showing the severity of chemotherapy mostly include the patients' mortality as study endpoints [2].

It is vital to assess the severity of different cancers separately as the disease biology varies and may exert differential effects on the immune system. Here we present our observations in five cancer-COVID admitted to Odisha COVID hospital $(\mathrm{OCH})$, managed patients by Kalinga Institute of Medical Sciences (KIMS), Bhubaneswar,

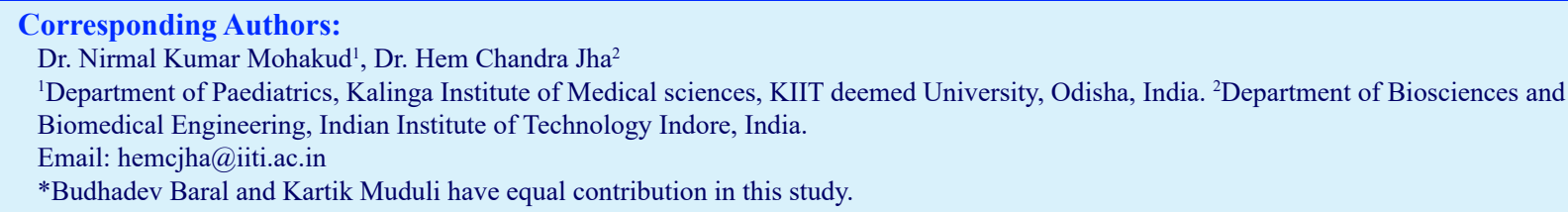


Odisha, India. We have also demonstrated the effect of COVID-19, cancer and its therapy on organs like the liver and kidney.

\section{Case presentation}

\section{Case 1}

A 62-year female was admitted as an RT-PCR confirmed SARS-CoV-2 positive case with a chief complaint of shortness of breath. She was suffering from carcinoma of the stomach and cervix with lung and brain metastasis. The patient had hypoxia at the time of admission hence kept in the ICU on oxygen support. On the day of admission, she was given a dose of paracetamol $650 \mathrm{mg}$ and Azithromycin $500 \mathrm{mg}$. Samples were also sent for routine hematological and biochemical tests. The reports suggested lower levels of sodium and potassium (Table 1). The CRP (C-reactive Protein) and procalcitonin were increased by approximately 16-fold, showing high infection levels. The hematological parameters related to RBC and haemoglobin (hematocrit, $\mathrm{MCH}, \mathrm{MCV}$ and RDW-CV) were also deregulated (Table 1). The patient had thrombocytopenia, lymphopenia and neutrophilia, showing characteristics of COVID-19. The level of urea and creatine (kidney function test) was marginally increased (Table 1).

Liver function test (LFT) was normal except for total protein, which decreased to $5.5 \mathrm{gm} / \mathrm{dL}$ (Table 1). After assessing the reports, Ivermectin $12 \mathrm{mg}$ was also given to reduce the viral load on the day 3 of the admission. Antiinflammatory (Dexamethasone $0.5 \mathrm{mg}$ ), antibiotic (Cefepime $2000 \mathrm{mg}+$ Tazobactum $250 \mathrm{mg}$ ) and anticoagulant medication (Enoxaparin $40 \mathrm{mg}$ ) were also given in view of the critical condition and high level of infection. Despite all the medications the patient had persistent hypoxia, for which she was managed with continuous oxygen support. On the $14^{\text {th }}$ day of admission, she developed respiratory distress and was kept of Non-invasive ventilation (NIV) support. In the early hours of day 15, she developed bradycardia, for which resuscitation was tried. Despite all the efforts, the patient could not be revived and was declared dead.

\section{Case 2}

A 70-year male patient was referred to the hospital from Acharya Harihar regional cancer center, Cuttack, as a SARS-CoV-2 positive case. He was suffering from cancer of the bladder with metastasis and had hypoxia at the time of admission, kept on oxygen support. Samples were sent for routine biochemical, hematological tests. A dose of paracetamol $650 \mathrm{mg}$ and Azithromycin $500 \mathrm{mg}$ along with vitamin c supplements were also given. The test reports suggested deregulated hematological parameters (Table 1). Specifically, the parameters related to RBC and hemoglobin were highly deregulated due to continuous blood loss. He was given Tranexamic acid $500 \mathrm{mg}$ tablets to stop the bleeding.

Additionally, Ferrous Ascorbate (Iron), Folic acid, Methylcobalamin, and Zinc supplements were also given. The serum sodium level was decreased to $128 \mathrm{mmol} / \mathrm{L}$ hence administered with $\mathrm{NaCl}$ solution. The urea $(208 \mathrm{mg} / \mathrm{dL})$ and creatinine $(4.48 \mathrm{mg} / \mathrm{dL})$ levels were also high, suggesting an impaired kidney function. The patient was having inflammation and pain in the urethra hence given Lignocaine 2\% jelly. Further, the inflammatory markers like CRP and procalcitonin were approximately 70 and 1200-fold higher than the normal range, respectively. The patient had lymphopenia and neutrophilia, showing characteristics of COVID-19. The patient's CT thorax showed calcified nodule and atelectatic changes in the right middle lobe and pleural thickening with thin fibrotic strands in the apical segment of the bilateral upper lobe. The severity score was $4 / 25$. On the $3^{\text {rd }}$ day of hospitalization, the patient developed respiratory distress and shifted to ICU and initially continued on NIV, later on mechanical ventilation (MV) support. Despite all resuscitative measures patient had persistent hypoxia. On the $6^{\text {th }}$ day of hospitalization, he developed bradycardia for which resuscitative measures were taken. Despite all effort patient could not be revived and was declared dead.

\section{Case-3}

A 62-year female patient was admitted to the hospital as a SARS-CoV-2 positive case with symptoms of fever and cough for last 7-days. She was having high-grade glioma and was hypoxic at the time of admission, for which she was kept in ICU. On admission blood samples were sent for routine tests. On the same day she was given a dose of Paracetamol $650 \mathrm{mg}$ and Azithromycin $500 \mathrm{mg}$. The report suggested slightly deregulated hematological and biochemical parameters (Table 1). The CT thorax was also showing few atelectatic changes with a severity score of $1 / 25$. On day two she was given Hydroxychloroquine 200 $\mathrm{mg}$ tablet and administered with Dexamethasone $4 \mathrm{mg}$ and Ascorbicacid $1.5 \mathrm{mg}$ injection. She was given Ivermectin tablet along with Dexamethasone $4 \mathrm{mg}$ injection on the $4^{\text {th }}$ day of hospitalization.

Further, she developed respiratory distress for which she was given NIV initially and later MV support. The patient deteriorated despite supportive treatment and her condition remained critical. On the $7^{\text {th }}$ day of hospitalization, she developed bradycardia. Resuscitation was done but despite all effort patient could not be revived and was declared dead.

\section{Case-4}

A 57-year male patient was on chemotherapy (Cisplatin and docetaxel) for his carcinoma of left lung when he developed symptoms like cough and fever. $\mathrm{He}$ was found positive for SARS-CoV-2 and started developing symptoms of breathlessness. The patient got admitted for supportive management of his symptoms and shifted to ICU in view of deteriorating symptoms. Samples were sent for routine biochemical and hematological tests. On the day of admission, the patient was given Paracetamol $650 \mathrm{mg}$ and Azithromycin $500 \mathrm{mg}$ and administered with Dexamethasone $4 \mathrm{mg}$, Enoxaparin $40 \mathrm{mg}$ and Cefepime $2000 \mathrm{mg}+$ Tazobactum $250 \mathrm{mg}$. The test report suggested deregulated hematological parameters and increased level of inflammatory markers (Table 1). The phosphorous 
Table 1. Haematological, Biochemical, Demographic Data of Cancer-COVID Patients. (Bold letters- Not in Normal Range, NA- Data not available)

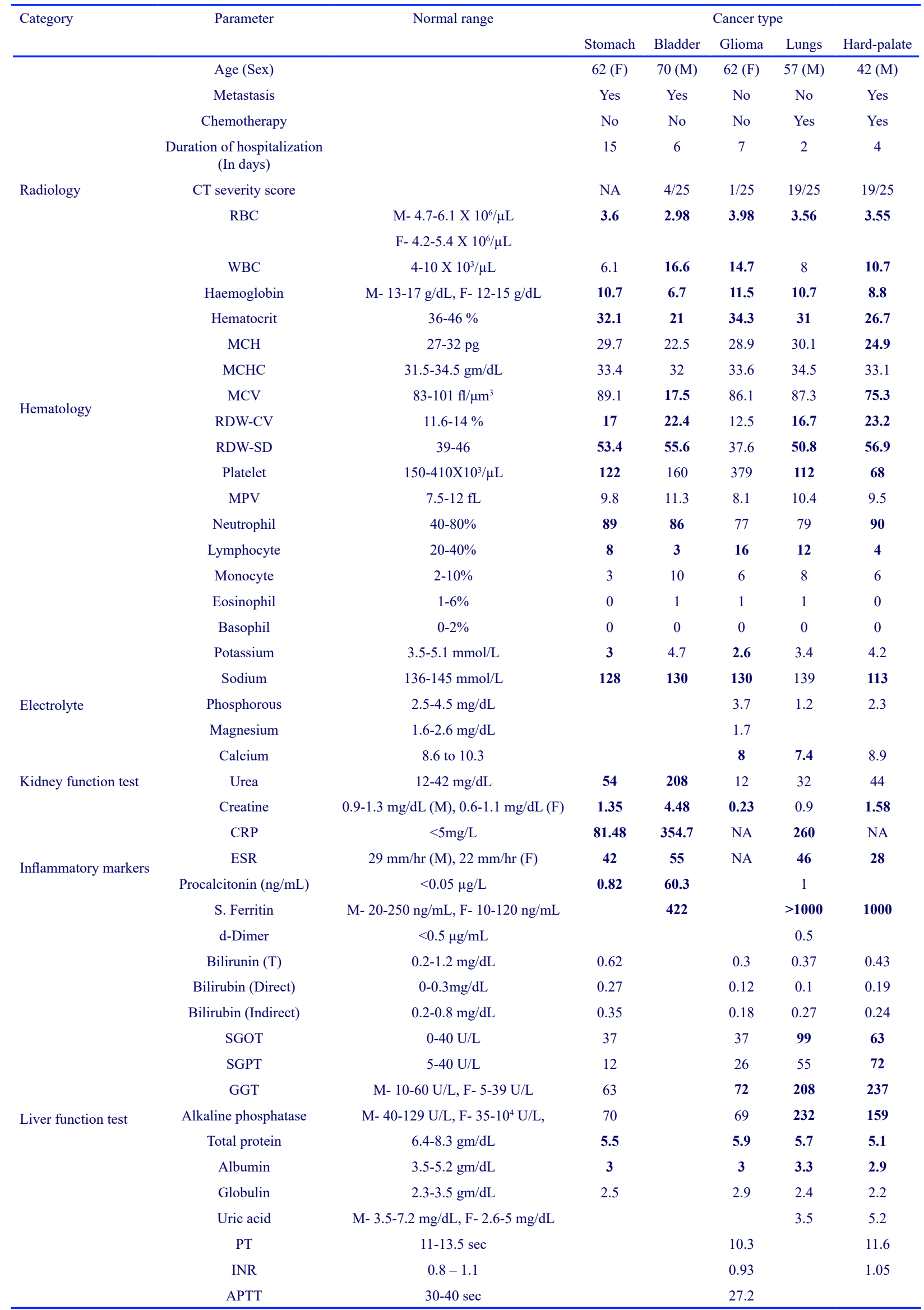


(A)

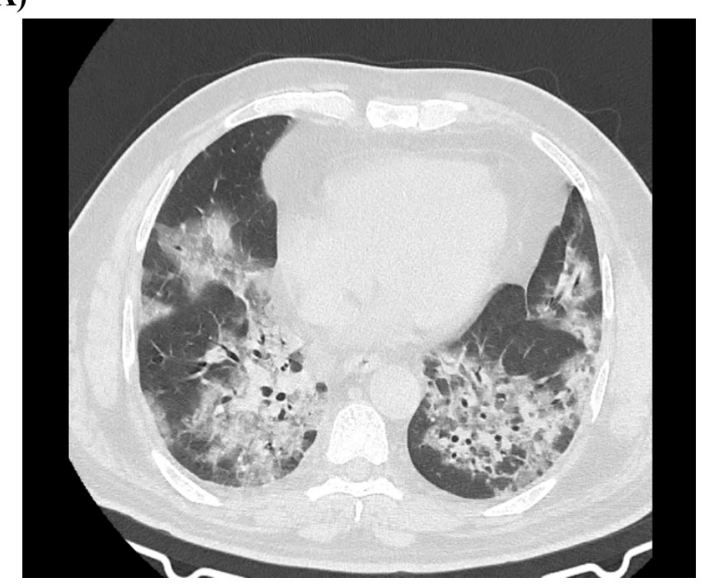

(B)

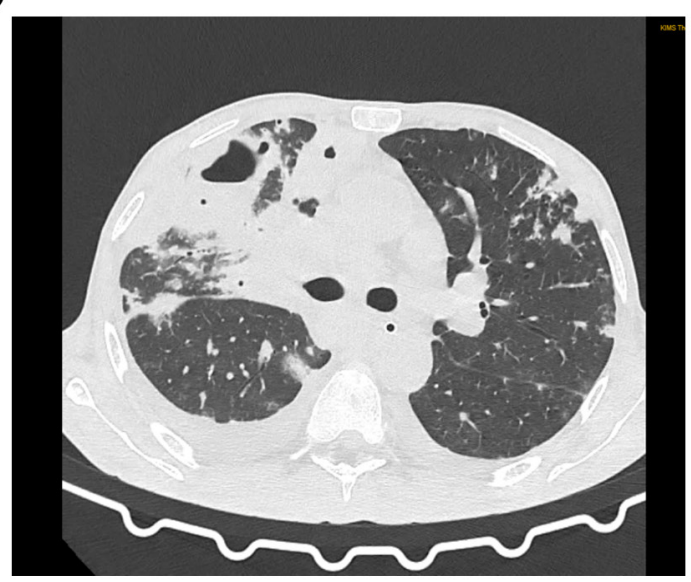

Figure 1. CT Thorax of Cancer-COVID Patients. The representative CT image of Cancer-COVID patients. (A) CT thorax of patient 4 (lung cancer). The image showing Bilateral peripheral and centrilobular ground glass opacities with consolidation. (B) CT thorax of patient 5 (cancer of hard palate). The image showing left sided peripheral ground glass opacities, right lung- consolidation with cavitation, interseptal and pleural effusion in addition to ground glass opacities. Both these patients were receiving chemotherapy at the time of infection and had CT severity score of $19 / 25$.

and calcium level was also low (Table 1). Notably, the LFT parameters like serum glutamic oxaloacetic transaminase (SGOT), serum Glutamate pyruvate transaminase (SGPT), gamma-glutamyl transpeptidase (GGT) and alkaline phosphatase was highly increased (Table 1). Further, thorax CT showed diffuse ground glass opacities with interlobular septal thickening (crazy paving) in all the lobes of both lungs predominantly in bilateral lower lobes (Figure 1 A). The CT severity score was $19 / 25$. On the $2^{\text {nd }}$ day of hospitalization, the patient was again administered with Dexamethasone $4 \mathrm{mg}$ and Cefepime $2000 \mathrm{mg}+$ Tazobactum $250 \mathrm{mg}$. Despite all the efforts, the condition of the patients remained grim and patient started developing bradycardia. CPR started as per the ATLS protocol and the patient was given $1 \mathrm{mg} / \mathrm{mL}$ adrenaline and atropine. The patient could not be revived and was declared dead.

\section{Case-5}

A 42-year male was admitted to the hospital as a case of Severe Acute Respiratory Infection (SARI) and COVID-19. The patient also had cancer of the hard palate with metastasis. The patient was on chemoradiation therapy when he got infected. Samples were sent for routine tests on the same day and treatment was started with Paracetamol $650 \mathrm{mg}$ and Azithromycin $500 \mathrm{mg}$ tablets along with vitamin-C supplements. Reports suggested deregulated hematological parameters (Table 1). The level of sodium and phosphorous was also lower than the normal range (Table 1). Importantly, SGPT, SGOT, GGT and alkaline phosphatase were highly increased, showing a probable malfunction in the liver (Table 1). The patient's chest $\mathrm{CT}$ was showing mild right and left minimal pleural effusion with the collapse of the underlying lung (Figure $1 \mathrm{~B}$ ). The CT severity score was $19 / 25$. On day 3 of hospitalization, the patient had an episode of bleeding (approximately $200 \mathrm{ml}$ ) from the oral cavity for which he was injected with Tranexamic acid $500 \mathrm{mg}$, Tranemic $500 \mathrm{mg}$ and shifted to ICU. The patient started developing respiratory depression and later kept on NIV for the same. On the $4^{\text {th }}$ day of hospitalization, he had an episode of bradycardia. Resuscitation tried and despite all efforts and resuscitative measures patient could not be revived and was declared dead.

\section{Discussion}

The consequence of COVID-19 in cancer patients were reported in previous studies [2]. However, it had not been studied together in different cancer types. In this case series, we reported determinants of severity in the included cases and the effect of cancer treatment on severity.

Patient- 4 and 5 died two and four days after hospitalization respectively. Both of these patients underwent chemotherapy when they acquired the infection. A study on the severity of COVID-19 in lung cancer patients showed that the patients undergoing oncotherapy have higher mortality than otherwise [4]. However, Lee et al. reported no significant difference in severity and mortality between oncotherapy recipients and nonrecipients [2]. Although studies showing opposite patterns are available these may further depend on the course of infection, organs affected by cancer and whether cancer is metastatic besides the treatment. Further, the parameters related to iron physiology were more deranged in patient- 2 and 5 . The iron deficiency in patient- 2 might be due to older age.

Additionally, patient-5 had an episode of bleeding and was also receiving chemoradiation therapy. Previous reports have suggested that increased pro-inflammatory cytokines like IL- 6 , IL- 1 , TNF- $\alpha$, interferon- $\gamma$, and antiinflammatory cytokine like IL-10 can affect the iron physiology and can cause functional iron deficiency 
[5]. Some or all of these cytokines are also known to be elevated during COVID-19 and most cancers.

Electrolytes are physiologically important and their deregulation can lead to complications. Low levels of sodium, potassium and calcium are associated with COVID-19 severity. However, in our study hypokalaemia was found in patient- 1 and 3 only. A lower level of calcium and phosphorus in patient-4 might be due to SARS-CoV-2 infection. A previous report suggested that serum calcium and phosphorus significantly decreased in infections like pulmonary tuberculosis [6].

The highly increased urea and creatinine level in case of patient-2 might be due to impaired kidney function. Previous studies have suggested impaired KFT as a risk factor for developing bladder cancer [7]. CRP and ESR (Erythrocyte Sedimentation Rate), broad-spectrum inflammatory markers, are known to be elevated in both COVID-19 and cancer. We also got a similar pattern of inflammatory markers. Both CRP and ESR were highest in the patient-2; this might be due to a synergistic effect of COVID-19, metastatic cancer and aging.

Lymphopenia is associated with COVID-19 and cancer. In our study, all patients had developed lymphopenia. Further, the thrombocytes show contradictory patterns in cancer and COVID-19 [8, 9]. In our study, platelet count did not fall drastically in any of the patients. The higher platelet count and almost normal lymphocyte count in glioma can be attributed to Isocitrate dehydrogenase 1 (IDH1) mutation, which decreases inflammation [10].

The liver plays a vital role in immunity and metabolism. In COVID-19 patients, mildly abnormal serum LFTs are common [11]. Further, patients undergoing chemotherapy have impaired liver function due to the hepatotoxic effect of chemotherapeutic drugs [12]. In the current study, the patients receiving chemotherapy had increased LFT enzymes like SGOT, SGPT, GGT and alkaline phosphatase. Thus, showing a possible hazardous effect of cancer chemotherapy and COVID-19. Importantly these patients had a higher CT severity score than those not receiving chemotherapy.

The report showed a vital association of hepatic enzyme elevation in cancer-COVID patients receiving chemotherapy. These patients are vulnerable and should be treated with caution. Adjuvant or palliative chemotherapy in patients with cancer should be carefully weighed against risks and discussed in Institutional Tumor Boards before administration, considering the severity in those patients. Patients in need of palliative care and poor functional status might not benefit from it in the current pandemic scenario. However, adjuvant chemotherapy can be considered in otherwise fit patients without significant comorbidities, with the understanding that hepatic dysfunction of varying severity might ensue, requiring cessation of chemotherapy in patients with severe enzyme elevation.

The glioma patient had low inflammation levels plausibly due to IDH1 mutation; however, we did not find any positive outcome for this patient's disease. Ageing is a great contributing factor in disease severity in cancer-COVID patients. It would be interesting to determine whether the outcomes in primary brain tumor patients with COVID-19 infection are similar to that of other patients without primary brain tumour. If so, this subset of patients with good functional status might continue with unmodified treatment, even during the pandemic.

Since the primary organ affected by COVID-19 is the lungs, it would be expected to have serious consequences for lung cancer patients with COVID-19 infection. These patients need to be looked at closely in future studies.

Our study includes a small number of patients hence might not be conclusive. The patients belong to a particular region (Odisha, India); thus, the manifestations may vary regionally. Most of the parameters we measured only once hence might have changed during the course of the treatment. Further data for certain parameters were not available for all the patients.

Each cancer has a different biology and a different impact on the internal milieu of the patient. COVID-19 infection and the cytokines released by the infection are likely to interact differently with the cytokines and other molecules released in higher levels as a result of cancer. This interaction can have a different outcome in different cancer types and thus impact the severity of illness, morbidity and mortality.

Despite the limitations, this study reported important findings on cancer-COVID patients and further studies (retrospective and prospective). Large populations are warranted to establish the findings comprehensively and identify subsets of cancer patients who might be more vulnerable during the COVID-19 pandemic.

\section{Ethics statement}

The ethical committees approved the protocol for the present study of the Indian Institute of Technology Indore, Indore (BSBE/IITI/IHEC-05/2020); School of Biotechnology, Kalinga Institute of Industrial Technology, Bhubaneshwar (KIIDU/KSBT/2020/345); and Kalinga Institute of Medical Sciences, Bhubaneshwar (KIIT/KIMS/IEC/372/2020). All procedures were performed in accordance to the revised declaration of Helsinki. Written consent was obtained from patient's family member.

\section{Acknowledgments}

We gratefully acknowledge the Kalinga Institute of Medical Sciences, Bhubaneshwar; School of Biotechnology, Kalinga Institute of Industrial Technology, Bhubaneshwar; and Indian Institute of Technology Indore to provide facilities support. We acknowledge the Council for Scientific and Industrial Research and the Department of Science and Technology Govt. of India for funding. We are also thankful to University Grants Commission, Govt. of India for fellowship to Budhadev Baral and Kartik Muduli in the form of research stipend. We appreciate lab colleagues for insightful discussions and advice. 


\section{References}

1. Tian J, Yuan X, Xiao J, Zhong Q, Yang C, Liu B, Cai Y, Lu Z, Wang J, Wang Y, Liu S, Cheng B, Wang J, Zhang M, Wang L, Niu S, Yao Z, Deng X, Zhou F, Wei W, Li Q, Chen X, Chen W, Yang Q, Wu S, Fan J, Shu B, Hu Z, Wang S, Yang X, Liu W, Miao X, Wang Z. Clinical characteristics and risk factors associated with COVID-19 disease severity in patients with cancer in Wuhan, China: a multicentre, retrospective, cohort study. The Lancet Oncology. 2020 07;21(7):893-903. https:// doi.org/10.1016/s1470-2045(20)30309-0

2. Lee LY, Cazier J, Angelis V, Arnold R, Bisht V, Campton NA, Chackathayil J, Cheng VW, Curley HM, Fittall MW, Freeman-Mills L, Gennatas S, Goel A, Hartley S, Hughes DJ, Kerr D, Lee AJ, Lee RJ, McGrath SE, Middleton CP, Murugaesu N, Newsom-Davis T, Okines AF, Olsson-Brown AC, Palles C, Pan Y, Pettengell R, Powles T, Protheroe EA, Purshouse K, Sharma-Oates A, Sivakumar S, Smith AJ, Starkey T, Turnbull CD, Várnai C, Yousaf N, Kerr R, Middleton G. COVID-19 mortality in patients with cancer on chemotherapy or other anticancer treatments: a prospective cohort study. The Lancet. 2020 06;395(10241):1919-1926. https://doi.org/10.1016/s0140-6736(20)31173-9

3. Jakhmola S, Indari O, Baral B, Kashyap D, Varshney N, Das A, Chatterjee S, Jha HC. Comorbidity Assessment Is Essential During COVID-19 Treatment. Frontiers in Physiology. 2020 08 04;11. https://doi.org/10.3389/fphys.2020.00984

4. Calles A, Aparicio MI, Alva M, Bringas M, Gutierrez N, Soto J, Arregui M, Tirado VC, Álvarez EL, del Monte-Millán M, Massarrah T, Galera M, Álvarez R, Martín M. Outcomes of COVID-19 in Patients With Lung Cancer Treated in a Tertiary Hospital in Madrid. Frontiers in Oncology. 202009 16;10. https://doi.org/10.3389/fonc.2020.01777

5. Ludwig H, Evstatiev R, Kornek G, Aapro M, Bauernhofer T, Buxhofer-Ausch V, Fridrik M, Geissler D, Geissler K, Gisslinger H, Koller E, Kopetzky G, Lang A, Rumpold H, Steurer M, Kamali H, Link H. Iron metabolism and iron supplementation in cancer patients. Wiener klinische Wochenschrift. 201509 15;127(23-24):907-919. https://doi. org/10.1007/s00508-015-0842-3

6. Rohini K, Bhat S, Srikumar PS, Mahesh Kumar A. Assessment of Serum Calcium and Phosphorus in Pulmonary Tuberculosis Patients Before, During and After Chemotherapy. Indian Journal of Clinical Biochemistry. 2013 Oct 11;29(3):377-381. https://doi.org/10.1007/s12291013-0383-3

7. Malyszko J, Tesarova P, Capasso G, Capasso A. The link between kidney disease and cancer: complications and treatment. The Lancet. 2020 07;396(10246):277-287. https:// doi.org/10.1016/s0140-6736(20)30540-7

8. Baranyai Z, Jósa V, Tóth A, Szilasi Z, Tihanyi B, Zaránd A, Harsanyi L, Szállási Z. Paraneoplastic thrombocytosis in gastrointestinal cancer. Platelets. 201605 02;27(4):269-275. https://doi.org/10.3109/09537104.2016.1170112

9. Lippi G, Plebani M, Henry BM. Thrombocytopenia is associated with severe coronavirus disease 2019 (COVID-19) infections: A meta-analysis. Clinica Chimica Acta. 2020 07;506:145-148. https://doi.org/10.1016/j. cca.2020.03.022

10. Huang J, Yu J, Tu L, Huang N, Li H, Luo Y. Isocitrate Dehydrogenase Mutations in Glioma: From Basic Discovery to Therapeutics Development. Frontiers in Oncology. 2019 06 12;9. https://doi.org/10.3389/fonc.2019.00506

11. Bertolini A, Peppel IP, Bodewes FA, Moshage H, Fantin A, Farinati F, Fiorotto R, Jonker JW, Strazzabosco M, Verkade HJ, Peserico G. Abnormal Liver Function Tests in Patients
With COVID-19 : Relevance and Potential Pathogenesis. Hepatology. 2020 Oct 20;72(5):1864-1872. https://doi. org/10.1002/hep.31480

12. Field KM, Dow C, Michael M. Part I: Liver function in oncology: biochemistry and beyond. The Lancet Oncology. 2008 Nov;9(11):1092-1101. https://doi.org/10.1016/s14702045(08)70279-1

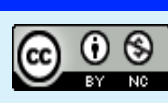

This work is licensed under a Creative Commons AttributionNon Commercial 4.0 International License. 